\title{
An Examination of the Influence of Big Five Personality on Service Orientation of University Students
}

\author{
Qingjuan WANG ${ }^{1, a,{ }^{*}}$, Jing $\mathrm{LI}^{2, \mathrm{~b}}$ and Qing Fan $^{3, \mathrm{c}}$ \\ ${ }^{1,2,3}$ College of Tourism and Service Management, Nankai University, Tianjin, P.R. China \\ a'wqingjuan@nankai.edu.cn, ${ }^{\mathrm{b}}$ nklijing911010@mail.nankai.edu.cn, cfqing@nankai.edu.cn
}

\begin{abstract}
Based on the theory of big five personality and service orientation, the current paper explored the effect of big five personality on service orientation and of service orientation on helping behavior of university students, presented a conceptual model regarding how big five personality affects service orientation of university students. In the model, agreeableness, extroversion, openness to experience, and conscientiousness predict service orientation of university students positively, while neuroticism predicts service orientation of university students negatively; Moreover, service orientation leads to helping behavior, students with high service orientation are more willing to help others. The implications and future research directions are discussed finally.
\end{abstract}

Keywords: Service orientation, big five personality, helping behavior, university students.

\author{
大五人格影响高校大学生服务导向的研究 \\ 王庆娟 ${ }^{1, a,{ }^{*}}$, 李晶 ${ }^{2, b}$, 范清 ${ }^{3, c}$ \\ $1,2,3$ 南开大学旅游与服务学院, 天津, 中国

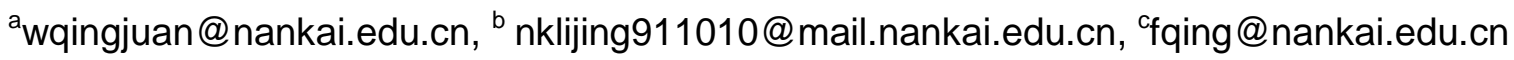

中文摘要. 本文以大五人格和服务导向为 理论基础, 通过文献回顾和理论分析探讨了 大五人格对于大学生服务导向的影响以及 服务导向对帮助行为的影响, 提出了大五人 格影响高校大学生服务导向的概念模型。模 型中，宜人性、外倾性、开放性和责任心正 向预测了高校大学生的服务导向, 神经质负 向预测了高校大学生的服务导向; 同时, 服 务导向进一步导致帮助行为, 具备高服务导 向的大学生更加乐于助人。文章最后讨论了 研究的意义和未来研究方向。

关键词: 服务导向; 大五人格; 帮助行为; 大学生

\section{1. 引言}

伴随产业结构的升级和转型, 世界上诸 多国家已经或正在将其经济重心转移至服 务业。这种趋势改变了以往企业仅通过产品 差异化吸引顾客注意力从而增强竞争优势 的传统态势, 取而代之的是强调通过优质服 务满足顾客差异化需求并最终实现顾客忠 诚和组织有效性的经营战略。在此背景下, 具备服务导向的人力资源成为企业获得成 功的关键要素之一。

从个体层面讲, 服务导向一方面源自于 个体稳定的人格特质，另一方面受制于后天 的经历以及成长环境。当前国内外学者通常 从组织氛围、组织授权等情境因素研究个体 的服务导向, 忽略了人格特质等个体层面因 
素对服务导向的影响。另外，目前服务导向 的研究对象主要集中于企业一线服务员工, 少有研究关注作为一线服务员工来源的高 校大学生群体; 相关研究主要集中在如何培 养改善现有员工服务导向的后觉问题上, 而 忽略了培养大学生服务导向和甄选具有服 务导向的大学生员工的先觉问题。因此, 本 研究的目的是通过文献回顾和理论分析, 探 讨大五人格对服务导向的预测作用以及服 务导向对于帮助行为 (组织公民行为的核心 维度之一) 的影响, 提出大五人格预测高校 大学生服务导向的概念模型, 并就服务型人 才的培养与开发从个体、高校和企业层面提 出合理化建议。

\section{2. 大五人格}

“人格” 是构成一个人的思想、情感以 及行为的独特模式, 这个独特模式包含了一 个人区别于他人的稳定而统一的典型的心 理品质 [1]。人格具有独特性、稳定性、统 合性和功能性的特征。大五人格模型

(Five-Factor Model, FFM) 是重要而成熟的 人格特质理论之一。大五人格模型由外倾性 (Extraversion)、宜人性(Agreeableness)、责 任心(Conscientious)、神经质 (Neuroticism) 和开放性(Openness)五个维度构成。外倾性 主要体现个体的人际关系和社交程度, 表现 为积极乐观、友好热情、富于冒险精神、喜 爱交际等; 宜人性用于衡量个体对于他人的 接受和依从程度, 表现为相信他人、顺从和 谦虚等; 责任心体现个体对目标的专注程 度, 表现为富有毅力、坚持不解、井井有条、 谨慎细心等; 神经质主要表现为敌对、敏感、 冲动等负面情绪; 开放性主要表现为富有想 象力和创造力, 求新求异和情感丰富[2]。

近年来, 越来越多的学者开始将大五人 格模型应用于服务业的研究当中, 其中部分 研究专门考察了大学生群体的相关问题。服 务行业对于从业人员与顾客的人际交互具 有高标准的要求, 热情、友好、积极和乐观 是对于该行业员工的最基础的从业要求。研 究发现大五人格特质对于个体从事工作的 类型和绩效水平具有一定的预测作用。例 如, 研究显示, 外倾性和开放性的人格特质 对大学毕业生从事社交型且富有挑战性的
工作具有正向的预测作用 [3]。参考文献[4] 的研究表明外倾性很好地正向预测了国际 留学生在东道国建立社交和人际关系的绩 效水平, 宜人性、开放性则与调整自我适应 新环境以及克制抱怨的绩效维度显著正相 关, 情绪的稳定性 (神经质的反面) 和帮助 他人、与他人合作以及努力程度显著正相 关。研究还发现, 在将周边绩效划分为合作 行为和建言行为 (voice behavior) 的基础上, 责任感、外倾性、宜人性和情绪的稳定性可 以有效地正向预测合作行为和建言行为 [5]。

因此, 大五人格模型中的五个维度都有 利于提升个体维持良好的人际关系, 促进个 体积极主动地帮助他人, 表现出乐于奉献的 意识和合作行为。对于服务业而言, 未来准 备从事服务工作的大学生, 可能需要具备高 外倾性、高宜人性、高责任心、高开放性以 及低神经质的人格特质, 以利于其今后在工 作中更好的处理与顾客之间的人际关系, 通 过提供令顾客满意的服务产品来提升顾客 的满意度和忠诚度。

\section{3. 服务导向}

关于服务导向的内涵, 学者们主要基于 特质观和情景观两个角度进行了研究。基于 特质观的定义最初由Hogan, Hogan和Busch 于1984年提出。他们认为服务导向是个体员 工乐于助人、体贴周到和易于合作的人格倾 向, 这种人格倾向最终会影响员工和顾客的 互动质量[6]。很多学者之后基于人格特质 观对服务导向进行了探讨。研究结果发现, 仅仅根据人格特质研究服务导向是不恰当 的, 因为服务导向会受到后天其他情境因素 的影响, 因此学者们又提出了基于情境观的 服务导向。

其中, Brown, Mowen, Donavan和Licata 通过COSE模型 (Customer Orientation of Service Employees，服务员工的顾客导向） 重新界定了服务导向, 并且在此基础上提出 了服务导向的二维模型。他们认为 “服务导 向是个体在工作岗位中所表现出的满足顾 客需求的态度和倾向” (pp. 111), 这种态 度和倾向分别由愉悦度和需求度两个维度 构成[7]。愉悦度主要表现为员工喜欢和顾 
客进行人际互动的程度; 需求度则主要表现 为员工坚信自己能够满足顾客需求的程度。 本文认为服务导向是一种态度倾向, 这种倾 向一方面表现为个体能够积极主动识别他 人的需求, 并且乐于帮助他人、满足他人的 需求。另一方面是指个体通过积极主动帮助 他人, 最终不仅仅带给他人惊喜和愉悦, 同 时还能够获得自我满足感和自身价值实现 感。本文采用[7]关于服务导向的定义和维 度划分进行后续研究。

服务业的研究已经证明, 服务组织的成 功主要基于热情友善的组织气候和员工高 质量的服务传递两个方面的因素, 具有竞争 优势的企业往往在组织层面和个体层面都 具有强烈的服务导向 [8]。研究表明, 如果 服务企业希望提升市场份额和利润, 那么以 顾客为中心、以服务导向为关注焦点则十分 具有战略优势 [9]。以往关注组织自身而非 顾客、控制产品质量和内部管理体系的做法 已经不足以建立持久的竞争优势, 管理者们 已经意识到组织的首要任务是服务顾客, 服 务导向对于企业获得战略优势具有非常重 要的作用。

\section{4. 大五人格对服务导向的影响}

人格特质对于个体的态度和行为具有 潜移默化和深远持久的影响, 这种影响会伴 随个体出生而逐步产生, 且难以在短时间内 产生质的变化。作为情感密集型行业, 员工 和顾客高质量的交互过程是服务业发展的 动力, 个体员工传递的服务态度和行为对于 顾客感知的服务质量具有重要的影响。而服 务传递的外在情境不会一成不变, 服务产品 自身具备生产与消费同步性、易逝性等特征 [10], 在服务传递中, 很难保证服务过程的 连贯性和固定性。因此针对外在情境因素不 可预测的变化, 员工内在稳定的人格特质可 能会在更大程度上决定其是否具有服务导 向的态度和是否能够提供优质服务的行为。 积极主动、热情洋溢、情绪稳定、机智敏锐 的员工更可能表现出乐于助人、满足顾客需 求的态度倾向并通过相应的服务行为使顾 客获得良好的体验。

首先，从与他人交往的关系角度来看， 外倾性、宜人性和开放性程度较高的大学生
通常会更加积极友善, 热情周到, 富有想象 力和创造力, 在行为处事中更加谦和, 乐于 与他人合作，乐于并有能力解决他人的问 题、满足他人的需求。其次, 责任心较强的 大学生更加认真、负责并具有较高的目标导 向, 会更加关心他人的需求, 将自己的利益 放诸于他人利益之后, 并表现出对待他人认 真负责和乐于满足他人需求的态度倾向。最 后, 神经质较低的大学生在与人交往的过程 中往往会表现出波澜不惊的态度, 更加沉着 冷静, 能够控制自己的情绪, 更容易识别并 满足他人需求, 因而具有较高的服务导向。

现有零散研究的发现也支持大五人格 特质与服务导向之间的关系。参考文献[7] 以食品行业249位员工为样本, 探讨了大五 人格与员工服务导向之间的关系。研究发 现, 大五人格中的宜人性和情绪稳定性解释 了食品业员工服务导向 $39 \%$ 的变异。参考文 献[11]对百货店145位经理的研究发现, 核 心自我评价与经理们的服务导向显著正相 关。由于核心自我评价的内涵与大五人格的 各个维度存在高度的相关与重叠[12], 因此 可以推断大五人格可能与服务导向也密切 相关。关于人格影响大学生服务导的研究非 常有限, 但作为未来服务业员工的储备力 量, 我们推断大学生群体的人格特征和服务 导向的关系将呈现出与现有服务业员工相 似的模式。

由此, 提出以下命题:

命题 1 : 大五人格特质能够预测大学生 的服务导向。

命题1a：外倾性与服务导向正相关

命题 $1 b$ : 宜人性与服务导向正相关

命题1c: 开放性与服务导向正相关

命题1d：责任心与服务导向正相关

命题1e: 神经质与服务导向负相关

\section{5. 服务导向对帮助行为的影响}

组织公民行为的概念由Organ 正式提 出, 指员工自愿从事的、超出自身职责范围 以外的行为, 这些行为能够提升组织的有效 性, 但并不直接受到组织的奖酬[13]。Organ 认为, 组织公民行为包含五个维度, 分别是 乐于助人、责任意识、运动员精神、礼节和 公民美德。不同的学者对组织公民行为的维 
度有不同的划分, [14]基于对已有研究的理 论分析与回顾, 最终将组织公民行为总结为 7 个维度, 分别是帮助行为、运动员精神、 组织忠诚、组织遵从、个人主动性、公民美 德以及自我发展。服务业员工的角色具有跨 边界性, 其组织公民行为除了涵盖上述内容 之外, 还包括员工主动向外界宣传组织、树 立组织良好形象等行为[15]。由于帮助行为 在组织公民行为研究中的重要性和较高的 信度与效度, 本文主要关注服务导向对帮助 行为的影响。

帮助行为 (Helping Behavior) 指个体 超出自身职责之外主动帮助他人的行为 [14]。由于服务企业很难规定员工在服务传 递中的具体行为, 因此员工需要承担一些组 织规定以外的工作以满足顾客需求并提高 服务质量[15]。在与外部顾客的互动中, 员 工主动帮助那些工作任务繁重而分身乏术 的同事, 经验丰富的老员工主动教授新员工 服务知识和技能, 帮助顾客解决突发问题, 满足顾客的临时需求, 都可以使顾客获得良 好的服务体验。服务导向较高的员工认为顾 客至上, 喜欢为顾客提供优质服务并乐在其 中[7]。这意味着即使是超出自身职责之外 的工作任务, 只要能够让顾客拥有良好的服 务体验, 服务导向较高的员工也会主动承 担, 表现出对组织内部同事或组织外部顾客 的帮助行为。

除了企业员工, 组织公民行为的研究也 一直在关注大学生群体, 尤其是学生干部。 在大学生组织中, 学生们所表现出的帮助行 为, 比如, 主动帮助新同学、帮助学习工作 任务繁重的同学、开展多种多样的活动以丰 富班级生活、尽心尽力进行活动的组织协调 等, 在很大程度上属于组织公民行为中帮助 行为的范畴。类似于企业员工, 服务导向较 高的大学生会比较关注他人, 会以他人的需 求为重, 并以满足他人需求为乐。对于超出 自身的学习和工作职责之外的任务, 如果能 改善同学的学习和生活体验, 服务导向较强 的学生也会主动承担。

大学生作为一组特殊人群, 在学校中往 往不会受到功利目的的影响, 因此这部分群 体表现出的服务导向和帮助行为更具有研 究意义。关于大学生群体服务导向影响其组
织公民行为尤其是帮助行为的研究非常有 限, 但依据上述分析, 我们可以得出以下命 题:

命题2: 大学生的服务导向和帮助行为 正相关

命题 $2 \mathrm{a}$ : 愉悦度（Enjoyment）与帮助 行为正相关

命题 $2 b$ : 需求度 (Need) 与帮助行为 正相关

本研究的概念模型见图1。

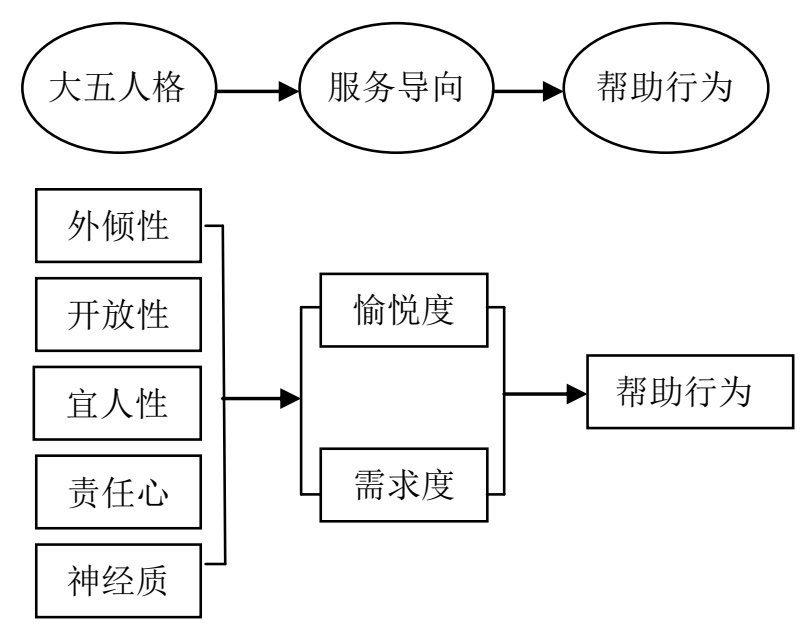

图1 概念模型

\section{6. 研究意义和未来研究方向}

本研究理论论证了大五人格影响大学 生服务导向以及服务导向影响帮助行为的 概念模型, 弥补了现有研究忽视个体层面因 素对服务导向的影响的不足, 同时增加了我 们对于大学生群体的人格特质影响服务导 向及帮助行为的理解, 具有重要的理论意 义。同时, 本研究还具有一定的实践价值。 首先, 组织就服务性工作在进行人才甄选 时, 可以将大五人格作为测评工具来预测应 聘者的服务导向, 从而判断应聘者是否适合 从事服务性工作, 更好地实现人职匹配。其 次, 高等院校可以对学生的人格特质进行测 评, 并通过系统化的课程和活动设计逐步改 善学生的人格特质, 提高其情绪的稳定性、 随和性、外倾性、开放性和责任心, 帮助学 生适应将来走入社会后可能要从事的服务 性工作。最后, 如果希望将来从事服务性工 作, 大学生应该提前对自己的人格特质进行 分析并进行有意识的培养、训练与改善。 
本研究也存在一定的不足。一方面, 本 研究为理论研究, 研究结论有待于进一步的 实证验证。未来研究可以选取合适的样本验 证本研究提出的概念模型。另一方面, 本研 究主要探究大五人格特质和服务导向的关 系, 但实际上, 高校大学生的服务导向同时 也会受到情境因素的影响, 因此就服务导向 的影响因素而言, 本研究的内容有待进一步 拓展。未来研究可以同时考虑情境因素和人 格特质对服务导向的影响, 系统全面地探讨 服务导向的决定因素。

\section{致谢}

本研究受中央高校基本科研业务费专 项资金项目（NKZXYY1119）、天津市哲 学社会科学基金规划项目（TJGL12-009） 资助。

\section{References}

[1] D. Funder. The Personality Puzzle. New York: Norton, 1997.

[2] R. R. Mccrae. O. P. John. An introduction to the five-factor model and its applications. Journal of Personality, vol. 60, pp.175-215, 1992.

[3] F. D. Fruvt and I. Mervielde. RIASEC types and big five traits as predictors of emplovment status and nature of emplovment. Personnel Psychology, vol.52, pp.701-727,1999.

[4] M. J. Burke. M. B. Watkins. and E. Guzman. Performing in a multi-cultural context: The role of personalitv. International Journal of International Relations, vol.33, pp. 475-485, 2009.

[5] J. A. LePine and L. Van Dvne. Voice and cooperative behavior as contrasting forms of contextual performance: Evidence of differential relationships with big five personality characteristics and cognitive ability, Journal of Applied Psychology, vol.86, pp.326-336, 2001.

[6] J. Hogan. R. Hogan. and C. M. Busch. How to measure service orientation,
Journal of Applied Psychology, vol.69, pp.167-173, 1984.

[7] T. J. Brown. J. C. Mowen. D. T. Donavan, and J. W. Licata. The customer orientation of service workers: Personality trait effects on self- and supervisor Performance Ratings. Journal of Marketing Research, vol.39, pp.110-119, 2002.

[8] A. Pizam. Service Orientation in the hospitality context. International Journal of Hospitality Management, vol.31, pp.1-2, 2012.

[9] E. Gummensson. Relationship marketing and imaginarv organizations: A svnthesis. European Journal of Marketing, vol.30, pp.31-44, 1996.

[10]E. Sasser. R. P. OLSEN. and D. D. Wvckoff. Management of Service Operations: Text. Cases and Readings. Boston: Allyn and Bacon , 1978.

[11]A. N. Salvaggio. B. Schneider. L. H. Nishi. D. M. Maver. A. Ramesh. and J. S. Lvon. Manager personalitv. manager service aualitv orientation and service climate. Journal of Applied Psychology, vol.29, pp.7141-7150, 2007.

[12]J. E. Bono and T. A. Judge. Core self-uations: A review of trait and its role in iob satisfaction and iob performance. European Journal of Personality, vol.17, pp.S5-S18, 2003.

[13]D. W. ORGAN. Organizational Citizenship Behavior: The Good Soldiers Svndrome. Lexington, MA: Lexington Books, 1988.

[14]P. M. Podsakoff. S. B. MacKenzie. J. B. Paine. and D. G. Bachrach. Organizational citizenship behaviors: A critical review of the theoretical and empirical literature and suggestions for future research. Journal of Management, vol.26, pp.513-563, 2000.

[15]L. A. Bettencourt. K. P. Gwinner. and M. L. Meuter. A comparison of attitude. personalitv. and knowledge predictors of service-oriented organizational citizenship behaviors. Journal of Applied Psychology, vol.86, pp.29-41, 2001. 